Vol. 16, Núm. 3

Jul.-Sep. 2020. pp 112-120 doi: $10.35366 / 94523$

https://dx.doi.org/10.35366/94523

\title{
Cirugía de cadera ante la pandemia COVID-19
}

\author{
Hip surgery in the face of the COVID-19 pandemic
}

\author{
Darío Esaú_Garín Zertuche, * José Alfredo_Penagos Paniagua, ${ }^{\ddagger}$ \\ Alfonso_Chávez Valenzuela, ${ }^{\S}$ Jonathan_Achoy Inzunzall
}

\begin{abstract}
* Cirujano Ortopedista. Egresado del Instituto Nacional de Rehabilitación-UNAM. Diplomado Artroscopia y Cirugía Articular. Subespecialidad en Reconstrucción Articular. Médico adscrito, Hospital Ángeles Tijuana. Profesor titular Curso Avanzado para Médicos Especialistas en Artroscopia y Reconstrucción Articular. ₹ Cirujano Ortopedista. Egresado de la Unidad Médica de alta Especialidad «Victorio de la Fuente Narváez", antes "Magdalena de las Salinas" IMSS. Fellow en Reemplazo articular y artroscopia de rodilla. Médico adscrito, Hospital General de zona Núm. 30 IMSS, Mexicali, Baja California.

$\S$ Cirujano Ortopedista. Egresado del Hospital Universitario de Puebla. Fellow Artroscopia y Reconstrucción Articular Hospital Ángeles Tijuana.

" Cirujano Ortopedista. Egresado del Hospital Civil de Culiacán. Fellow Artroscopia y Reconstrucción Articular Hospital Ángeles Tijuana.
\end{abstract}

Correspondencia: Dr. Darío Esaú Garín Zertuche. Paseo de los Héroes No. 10999-301, Zona Río, 22010, Tijuana, Baja California. Correo electrónico: dgarinmd@gmail.com

\section{RESUMEN}

La cirugía ortopédica electiva como reemplazos articulares primarios, revisión y artroscopias ha sido afectada por la pandemia. La mayoría de los pacientes que requieren cirugía de cadera son de edad avanzada, a excepción de los pacientes de artroscopia. Estos pacientes presentan comorbilidades con alteraciones en sus reservas funcionales y sistema inmunológico, por lo que son susceptibles a una infección por COVID-19. Se seleccionarán sólo a aquéllos cuya patología puede dejar secuelas importantes y poner en riesgo su bienestar físico. Las luxaciones e infecciones de cadera son consideradas como urgencias quirúrgicas debido a sus complicaciones al no ser tratadas, por lo que requieren atención dentro de las primeras ocho horas. Las fracturas de cadera son urgencias que requieren hospitalización y deben tratarse dentro de 24 a 48 horas. Para el tratamiento de fracturas intracapsulares por fragilidad ósea se recomienda la hemiartroplastia. En fracturas extracapsulares

\section{ABSTRACT}

Elective orthopedic surgery, primary articular replacements and arthroscopies have been affected by the pandemic. Most patients that require a hip surgery are from advanced age, with the exception of hip arthroscopy patients. These patients show comorbidities with alterations in their functional reserves and immune system by which they can be more severely affected by a COVID-19 infection, must be selected only the ones where the pathology could leave important sequels or risk their physical wellbeing. Dislocations and septic hip arthritis are considered surgical urgencies due to the complications that can present if they are not treated, by which they require attention within eight hours. Hip fractures are urgencies that require immediate hospitalization and must be treated within the first 24-48 hours. The treatment for intracapsular fractures by osseous fragility, hemiarthroplasty is recommended. For extracapsular fractures, 
se recomienda el uso de la placa tornillo deslizante en vez de clavo de reconstrucción por la disminución del tiempo quirúrgico y menor cantidad de instrumental. Las reanudaciones de cirugías electivas como artroscopias se realizarán cuando la situación pandémica se encuentre en su etapa de meseta, valorando la posibilidad de tener una estancia menor de 23 horas de hospitalización, siempre y cuando la unidad cuente con un área especializada para la atención y con ventiladores disponibles para cualquier eventualidad. Los reemplazos totales de cadera electivos se podrán realizar cuando el contagio máximo haya pasado y nuestra infraestructura de atención pueda soportar un nuevo aumento de infecciones por COVID-19.

Palabras clave: Cirugía de cadera, COVID-19, equipo de protección personal, medidas en quirófano. the use of dynamic hip screw instead of reconstruction nails because of the decrease in surgical time, amount of bleedings and less amount of instruments. Resumption of elective surgeries like arthroscopies will be made when the pandemic situation is in a stage of plateau, evaluating the possibility of having a hospital stay of less than 23 hours, as long as the unit has a specialized area to care for negative patients and count with available ventilators for any eventuality. Elective total hip replacements could be performed once the maximum contagion peak has passed and we are sure that our medical attention infrastructure can support a new rise in COVID-19 infections.

Keywords: Hip surgery, COVID-19, personal protection equipment, surgery measures.

\section{INTRODUCCIÓN}

El 08 de diciembre de 2019 aparece una neumonía de origen desconocido en Wuhan, provincia de Hubei, China. El 07 de enero de 2020, los científicos chinos confirman que el brote es causado por un nuevo coronavirus SARS-CoV-2. La OMS denomina a la enfermedad por este coronavirus COVID-19, la cual se disemina rápidamente de manera global, para más tarde declararla pandemia el 11 de marzo de 2020. ${ }^{1}$ La pandemia de COVID-19 ha afectado a la mayor parte del mundo y actualmente en Estados Unidos existen más de un millón de casos confirmados y a nivel mundial más de tres millones. En la actualidad en México se cuenta con 22,088 casos confirmados y 2,061 defunciones hasta el día 03 de mayo de $2020 .^{2}$

Aunque la ortopedia no está directamente relacionada con la atención de los pacientes afectados con COVID-19, ha experimentado la afección de la pandemia y los cambios en los sistemas de salud. Muchos ortopedistas en otros países han participado en el tratamiento de los pacientes menos graves con COVID-19 debido a la falta de recursos humanos y la gran cantidad de pacientes no ortopédicos en las unidades médicas. ${ }^{3}$

La cirugías ortopédicas electivas incluyendo los reemplazos articulares y la artroscopia de cadera han sido afectadas por la pandemia debido a que el uso de los recursos del sistema de salud se han centrado en el manejo de COVID-19, además de que no se puede garantizar la seguridad de los pacientes en tales situaciones, ya que existen limitaciones de personal, recursos y de equipos adecuados. Por estas razones, las cirugías con mayor riesgo quirúrgico y aquéllas consideradas como no urgentes deben ser reprogramadas. La cirugía ortopédica se considera de alta posibilidad de contaminación por la formación 
de aerosol y gotas de líquidos biológicos, por el uso de equipos de poder y por la cantidad de instrumental dentro de la sala de cirugía. ${ }^{4}$

Las artroplastias de cadera y rodilla son dos de los procedimientos electivos que más se realizan en Estados Unidos con alrededor de 1.5 millones de procedimientos anualmente, por lo que nos hemos visto obligados a rediseñar los procesos de atención. ${ }^{5}$

Se proyecta que este año se van a cancelar 481,771 reemplazos de cadera primaria y 1,078,359 reemplazos de rodilla primaria durante 2020 en EUA. ${ }^{6}$

\section{INDICACIONES PARA CIRUGÍA DE CADERA}

Debido a la pandemia COVID-19, la cirugía electiva de cadera será cancelada incluyendo la artroscopia y los reemplazos articulares primarios y de revisión. La mayoría de los pacientes que requieren de una cirugía de cadera son de edad avanzada, a excepción de los pacientes de artroscopia de cadera. Estos pacientes presentan comorbilidades con alteraciones en sus reservas funcionales y sistema inmunológico, por lo que pueden ser afectados más adversamente por una infección por COVID-19, motivo por el que deben seleccionarse sólo aquéllos cuya patología puede dejar secuelas importantes y poner en riesgo su bienestar físico.

Las luxaciones y artritis séptica de cadera son consideradas como urgencias quirúrgicas debido a las complicaciones que pueden traer al no ser tratadas, por lo que requieren su atención dentro de las primeras ocho horas. Las fracturas de cadera (intracapsulares y extracapsulares) son urgencias que requieren una hospitalización inmediata y deben tratarse dentro de las primeras 24 a 48 horas. ${ }^{7}$

\section{PREOPERATORIO CIRUGÍA DE CADERA}

\section{Protocolo para intervención de pacientes de patología quirúrgica de cadera}

Existen pacientes asintomáticos que son portadores del virus que están exponiendo involuntariamente a otros pacientes hospitalizados, ambulatorios y proveedores de atención médica al riesgo de contraer COVID-19.

Todos los pacientes sospechosos negativos que requieran intervención quirúrgica deben ser tratados como positivos hasta que se demuestre lo contrario para minimizar la propagación de la infección. En todo caso sospechoso o positivo hay que realizar una tele de tórax en busca de indicios radiográficos de consolidación pulmonar y si se cuenta con tomografía computarizada, ésta se puede utilizar para buscar apariencia de vidrio esmerilado multilobular ocasionada por los cambios pulmonares del COVID-19. ${ }^{8}$

Se realizan valoraciones para detectar COVID-19 dentro de las 72 horas previas a la cirugía, obteniendo resultados en menos de 24 horas. Las pruebas actuales incluyen: PCR de transcriptasa inversa y prueba de anticuerpos ELISA. A su ingreso los pacientes tendrán una verificación previa en un área específica (unidad respiratoria) para valorar síntomas similares a la influenza como fiebre, tos, disnea, malestar general. Se les realizará una encuesta donde se valorarán 
factores de riesgo como viajes recientes, familiares enfermos o exposición a COVID-19. Se recomienda la firma de formulario de consentimiento informado COVID-19 (FEMECOT). .,9 $^{8}$

Con uso de equipo de protección personal (EPP), incluyendo mascarilla quirúrgica, bata y guantes, el paciente debe presentarse solo, en caso de no ser posible se permitirá sólo un acompañante.

El personal debe mantener distanciamiento social (mínimo 1.5 metros de distancia cuando sea posible) y usar equipo de protección personal EPP (guantes, bata, mascarilla quirúrgica y gafas). Al ingreso del personal se recomienda realizar pruebas rápidas para detectar COVID-19 a su llegada o antes (una vez que las pruebas estén disponibles), tienen que estar capacitados para protegerse y proteger a los pacientes. Y por parte de las autoridades de las unidades otorgar medidas de apoyo para abordar la fatiga y la angustia emocional del personal.

Uso de antibióticos. Las cefalosporinas han sido los antimicrobianos preferidos durante los últimos 30 años, con éxito comprobado, para la profilaxis de la artroplastia de cadera, sin importar las afectaciones por COVID-19. ${ }^{10}$

Uso de anticoagulantes. Los pacientes con fractura de cadera se encuentran en alto riesgo de trombosis venosa profunda por el trauma y sus comorbilidades asociadas. ${ }^{11}$

Asimismo, los pacientes con COVID-19 presentan una hipercoagulabilidad junto con un severo estado inflamatorio, ocasionando embolia pulmonar y/o trombosis venosa profunda de las extremidades inferiores, por lo que se recomienda la profilaxis antitrombótica. ${ }^{12}$

Es aconsejable el uso de enoxaparina o fondaparinux a dosis indicadas para la profilaxis del tromboembolismo venoso en todos los pacientes COVID-19 hospitalizados; se debe administrar durante toda su estancia hospitalaria, continuando en casa durante 14-21 días después del alta, o en la fase prehospitalaria, en caso de que presentara factores de riesgo de trombosis venosa profunda y tromboembolia pulmonar. ${ }^{13}$

El traslado de pacientes hacia quirófano desde el área para pacientes con COVID-19 o sospecha debe ser sistemático con una ruta lo más directa y corta posible, lo más alejado del resto de pacientes y personas en general del hospital para minimizar los riesgos de infección. Cualquier paciente no intubado debe usar una máscara quirúrgica.

\section{TRANSOPERATORIO-CIRUGÍA}

Las áreas operativas de COVID-19 deben asignarse a las operaciones urgentes de los pacientes con esta patología. El quirófano debe ser el más cercano a la entrada y de uso exclusivo para pacientes con COVID-19. La sala de operaciones debe contar con presión negativa que sería lo ideal para minimizar el riesgo de infección, o en su caso presión positiva, pero con aire acondicionado apagado. El equipo que se encuentra en cada quirófano debe ser el mínimo de acuerdo a lo estrictamente necesario caso por caso. 
Una vez que la cirugía comienza, el personal que transita dentro y fuera de éste debe ser el mínimo necesario, para minimizar el riesgo de infección se recomienda utilizar dispositivos de material desechable en general. Todos los operadores (cirujanos, anestesiólogos, enfermeras y técnicos) deben ingresar a la sala a tiempo, con el objetivo de minimizar el tiempo de cirugía y realizar el procedimiento con el menor personal posible. Una vez en el quirófano, no deberán retirarse hasta que el procedimiento quirúrgico se haya completado, y una vez fuera no deben volver a entrar. ${ }^{14}$

La puerta del quirófano debe mantenerse cerrada en todo momento y despejada, se deben colocar letreros de prohibición de entrada innecesaria a la habitación. El suministro de materiales al quirófano durante la cirugía debe ser sólo el esencial. Los cirujanos deben realizar la operación de preferencia con lo que está disponible en el quirófano una vez que comenzó la cirugía. Cualquier recuperación esencial del equipo necesario deberá hacerse mediante el personal fuera del quirófano. ${ }^{15}$

\section{Equipo de protección personal en quirófano}

Todo el personal que se encuentra dentro la cirugía debe contar con guantes quirúrgicos, un gorro quirúrgico resistente al agua, bata con mangas largas, mascarilla quirúrgica y careta para protección completa de la cara. En casos de posible caso de COVID-19 deben usarse respiradores buscando la manera de evitar el uso de equipo de poder, electrocauterio y equipos de irrigación pulsátil. ${ }^{16}$

Cubrebocas. Se recomienda utilizar mascarillas respiratorias N95, N99 (Figura 1) y N100 que proporcionan un factor de protección de 11.5 a 15.9 veces mayor que las mascarillas quirúrgicas convencionales, y son viables sólo por ocho horas. En caso de procedimiento con producción de aerosol se recomienda el uso de respiradores purificadores de aire motorizados. ${ }^{16}$

Protección ocular con gafas o caretas. Se deben proteger los ojos de gotas de líquidos corporales y así evitar una contaminación conjuntival. Por lo tanto, dentro de las medidas preventivas se debe incluir el uso sistemático de gafas y/o caretas que cubran los ojos y la piel periocular para todos los trabajadores de la salud presentes en

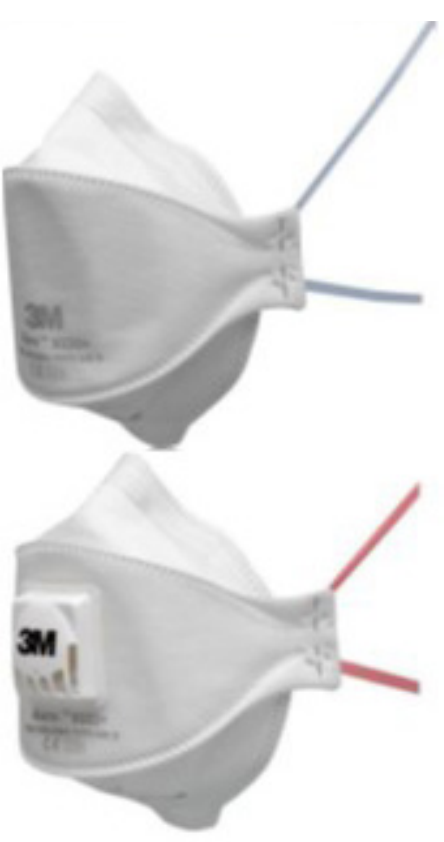

Figura 1: Máscara quirúrgica N95 (superior) y N99 (inferior). Copyright. ${ }^{16}$ 
la sala durante procedimientos potencialmente infecciosos generadores de aerosoles. ${ }^{16}$

Guantes quirúrgicos. Se recomienda el uso de guantes dobles debido a que disminuye el porcentaje de exposición sanguínea entre 10 y 54\%. También para reducir el riesgo de contaminación y perforación se recomienda aumentar las veces de cambio de guantes externos durante ciertas etapas quirúrgicas como incisión quirúrgica, colocación de implantes, cementación y reducción de prótesis. ${ }^{17,18}$

Anestesia. La anestesia neuroaxial y los bloqueos nerviosos periféricos son la primera opción (siem-
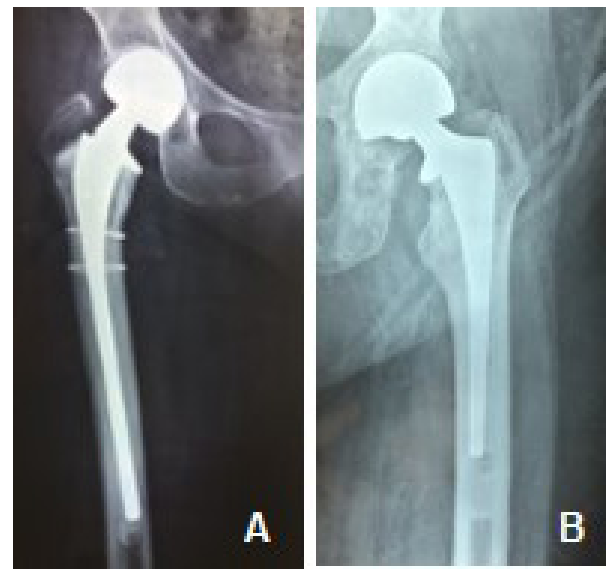

Figura 2: Hemiartroplastia de cadera A) derecha y B) izquierda. Cortesía: Dr. Darío Garín. pre que sea posible) para el manejo anestésico de pacientes con sospecha de infección por COVID-19, la cual debe realizarse en quirófano con EPP (guantes, bata, mascarilla quirúrgica y gafas $)^{19}$ con la menor cantidad posible de personal dentro de sala de quirófano. En caso de no ser posible y necesitar anestesia general se debe tener, igualmente, la cantidad mínima de personal que cuente con equipo completo de EPP, caja de vías respiratorias para protección, caja con medicamentos preparados y un video laringoscopio; la intubación endotraqueal será realizada por el anestesiólogo con más experiencia. ${ }^{20}$

Procedimiento quirúrgico. Se recomienda una cirugía lo menos traumática posible para evitar la menor exposición de tejido y fluidos al equipo quirúrgico, siempre y cuando el cirujano y su equipo tengan los conocimientos, habilidad e instrumental para realizarla.

La exposición e iluminación durante abordajes abiertos deben ser óptimas para facilitar el procedimiento y acortar, en lo posible, el tiempo de la intervención. Deben evitarse incisiones incómodas o demasiado pequeñas que añadan dificultad al procedimiento. Es aconsejable la utilización de separadores autoestáticos, pues no sólo facilitan la exposición del campo, sino que además minimizan la necesidad de ayudantes adicionales. ${ }^{21}$

Para el tratamiento de las fracturas intracapsulares por fragilidad ósea se recomienda la hemiartroplastia (Figura 2) en todas sus modalidades en vez de artroplastia total de cadera. En caso de fracturas extracapsulares se recomienda el uso de la placa tornillo deslizante (Figura 3) en vez de clavo de reconstrucción por la disminución del tiempo quirúrgico, cantidad de sangrado y menor cantidad de instrumental. ${ }^{21}$

El equipo de poder debe utilizarse lo menos posible así como el electrocauterio, el cual se recomienda con succión; para evitarse se debe contar con el material ortopédico indispensable y así producir menos cantidades de aerosol 
que contienen sangre y disminuir el riesgo para el paciente y el equipo quirúrgico de una infección por COVID-19. Teniendo en mente que el virus SARSCoV-2 puede durar hasta 72 horas en el acero, lo que aumenta el riesgo de contaminación, se recomienda utilizar material de osteosíntesis de otra composición en caso de contar con éste..$^{21,22}$

\section{MANEJO POSTQUIRÚRGICO}

Se recomienda realizar bloqueo regional posterior a las cirugías para valorar su pronto egreso y recuperación en casa con el fin de que el paciente se encuentre el menor tiempo expuesto a una infección por el virus; asimismo, ayudar a la disminución del uso de terapia con opioides que podrían suprimir el sistema inmunológico en la unidad. Debemos evitar la movilización de pacientes por la unidad para toma de Rayos $\mathrm{X}$ de control, por lo que se recomienda realizarlas con equipo portátil en quirófano.

Una vez egresado el paciente se darán indicaciones por escrito o por correo electrónico con el fin de no estar en contacto con el personal de salud. Los cuidados en su hogar incluyen seguimiento médico por llamada o videollamadas, rehabilitación en casa por telemedicina, las curaciones de heridas quirúrgicas no deben hacerse del diario, explicando previamente cómo realizarlas. ${ }^{23,24}$

\section{REGRESO A LA CIRUGÍA ELECTIVA}

Cuando la situación pandémica que nos está afectando se encuentre en su etapa de meseta, se podrá valorar la posibilidad de realizar procedimientos quirúrgicos electivos ambulatorios como artroscopias de cadera y retiro de materiales, los cuales podrían tener una estancia menor de 23 horas de hospitalización, siempre y cuando la unidad cuente con área especializada para atención a pacientes con COVID, con ventiladores disponibles para cualquier eventualidad y con el equipo necesario para protección tanto del paciente como del personal.

Los reemplazos totales de cadera electivos por artrosis se podrán realizar una vez que el punto de contagio máximo haya pasado y estemos seguros de que la infraestructura de atención médica pueda soportar un nuevo aumento de infecciones por COVID en nuestra población. ${ }^{23}$

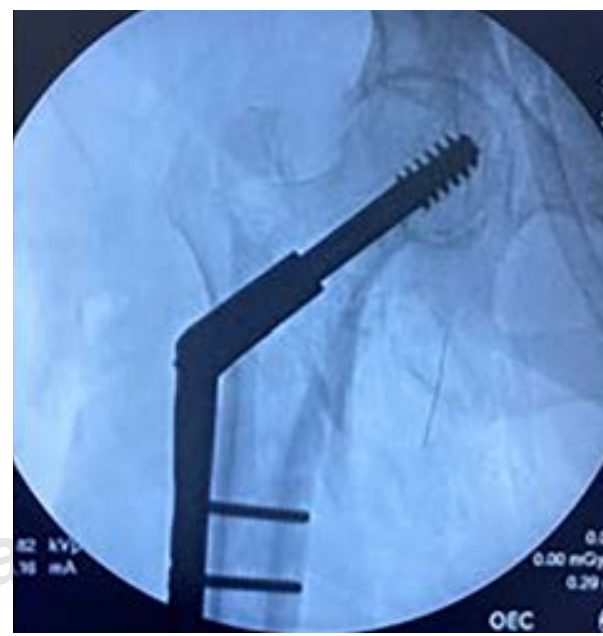

Figura 3: Placa tornillo deslizante. Cortesía: Dr. Darío Garín. 


\section{BIBLIOGRAFÍA}

1. Huang C, Wang Y, Li X, Ren L, Zhao J, Hu Y, et al. Clinical features of patients infected with 2019 novel coronavirus in Wuhan, China. Lancet. 2020; 395: 497-506.

2. Nuevo Coronavirus en el mundo COVID-19. Comunicado Técnico Diario. Gobierno de México, Secretaría de Salud. 02/05/2020. Disponible en: https://www.gob.mx/salud/es/archivo/prensa.

3. Khanuja HS, Chaudhry YP, Sheth NP, Oni JK, Parsley BS, Morrison JC. Humanitarian Needs: The Arthroplasty Community and the COVID-19 Pandemic. J Arthroplasty. 2020; S0883-5403(20)30441-1. Available in: https://www.arthroplastyjournal.org/action/showPdf?pii=S0883-5403\%2820\%2930441-1.

4. Rodríguez-Pinto R, Sousa R, Oliveira A. Preparing to perform trauma and orthopaedic surgery on patients with COVID-19. J Bone Joint Surg. 2020; 11: 946-950.

5. Brown TS, Bedard NA, Rojas EO, Anthony CA, Schwarzkopf R, Barnes CL, et al. The effect of the COVID-19 pandemic on electively scheduled hip and knee arthroplasty patients in the United States. $J$ Arthroplasty. 2020; S0883-5403(20)30439-3. Available in: https://www.arthroplastyjournal.org/action/ showPdf?pii=S0883-5403\%2820\%2930439-3.

6. Bedard NA, Elkins JM, Brown TS. Effect of COVID-19 on hip and knee arthroplasty surgical volume in the United States. J Arthroplasty. 2020; S0883-5403(20)30448-4. Available in: https://www.arthroplastyjournal.org/action/showPdf?pii=S0883-5403\%2820\%2930448-4.

7. American College of Surgeons COVID 19: Elective Case Triage Guidelines for Surgical Care. Adult elective Surgery and procedures Recommendations. [March 2, 2020]. Available in: https://www.facs. org/covid-19/clinical-guidance/elective-case.

8. Tang G, Ming-Chang AK. Perioperative management of suspected/ confirmed cases of COVID-19. General Anaesthesia Tutorial 421. [April 6, 2020]. Available in: https://www.wfsahq.org/components/ com_virtual_library/media/1c4ec5c64b9aaacf7c47f76a61fb6edc-atow-422-01.pdf.

9. Federación Mexicana de Colegios de Ortopedia y Traumatología A.C. Declaración de posición y recomendaciones FEMECOT ante la pandemia COVID-19. 11 de Abril 2020. Disponible en: https://www. femecot.com/webinar/covid-19/.

10. Meehan J, Jamali AA, Nguyen H. Prophylactic antibiotics in hip and knee arthroplasty. J Bone Joint Surg Am. 2009; 91 (10): 2480-2490.

11. Brill JB, Badiee J, Zander AL, Wallace JD, Lewis PR, Sise MJ, et al. The rate of deep vein thrombosis doubles in trauma patients with hypercoagulable thromboelastography. J Trauma Acute Care Surg. 2017; 83 (3): 413-419.

12. Panigada M, Bottino N, Tagliabue P. Hypercoagulability of COVID-19 patients in Intensive Care Unit. A report of thromboelastography findings and other parameters of hemostasis. J Thromb Haemost 2020. In press. Available in: https://onlinelibrary.wiley.com/doi/epdf/10.1111/jth.14850.

13. Marietta M, Ageno W, Artoni A, De Candia E, Gresele P, Marchetti M et al. COVID-19 and haemostasis: a position paper from Italian Society on Thrombosis and Haemostasis (SISET). Blood Transfus 2020. In press. Available in: http://www.bloodtransfusion.it/articolosing.aspx?id=001051.

14. Coccolini F, Perrone G, Chiarugi M, Di Marzo F, Ansaloni L, Scandroglio I, et al. Surgery in COVID-19 patients: operational directives. World J Emerg Surg. 2020; 15 (1): 25.

15. Wong J, Yuan GQ, Tan Z, An Lie S, Chuan TY, Yi NS, et al. Preparing for a COVID-19 pandemic: a review of operating room outbreak response measures in a large tertiary hospital in Singapore. Can J Anesth. 2020; 67: 732-745. Available in: https://www.ncbi.nlm.nih.gov/pmc/articles/PMC7090449/ pdf/12630_2020_Article_1620.pdf.

16. Hirschmann MT, Hart A, Henckel J, Sadoghi P, Seil R, Mouton C. COVID-19 coronavirus: recommended personal protective equipment for the orthopaedic and trauma surgeon. Knee Surg Sports Traumatol Arthrosc. 2020; 28 (6): 1690-1698. Available in: https://www.ncbi.nlm.nih.gov/pmc/articles/PMC7184806/ pdf/167 2020 Article 6022.pdf.

17. Beldame J, Lagrave B, Lievain L, Lefebvre B, Frebourg N, Dujardin F. Surgical glove bacterial contamination and perforation during total hip arthroplasty implantation: when gloves should be changed. Orthop Traumatol Surg Res. 2012; 98 (4): 432-440.

18. Thanni LO, Yinusa W. Incidence of glove failure during orthopedic operations and the protective effect of double gloves. J Natl Med. 2003; 95 (12): 1184-1188.

19. Uppal V, Sondekoppam RV, Landau R, El-Boghdadly K, Narouze S, Kalagara HK. Neuraxial anaesthesia and peripheral nerve blocks during the COVID-19 pandemic: a literature review and practice recommendations. Anaesthesia 2020. In press. Available in: https://onlinelibrary.wiley.com/doi/epdf/10.1111/ anae.15105.

20. Zucco L, Levy N, Ketchandji D, Aziz M, Krishna RS. Anesthesia Patient Safety Foundation. Recommendations for airway management in a patient with suspected coronavirus (2019-nCoV) infection. 
Available in: https://www.apsf.org/wp-content/uploads/news-updates/2020/apsf-coronavirus-airwaymanagement-infographic.pdf.

21. NHS UK. Specialty guides for patient management during the coronavirus pandemic Clinical guide for the perioperative care of people with fragility fractures during the coronavirus pandemic. [March 25, 2020]. Available in: https://www.england.nhs.uk/coronavirus/wp-content/uploads/sites/52/2020/03/ C0086_Specialty-guide-_Fragility-Fractures-and-Coronavirus-v1-26-March.pdf.

22. Brücher B, Nigri G, Tinêlli A, Lapeña JF Jr., Espin-Basany E, Macri P, et al. COVID-19: pandemic surgery guidance. 4open. 2020; 3: 1-19.

23. Kopp SL, Horlocker TT. Regional anaesthesia in day-stay and short-stay surgery. Anaesthesia. 2010; 65 (Suppl. 1): 84-96.

24. European Society of Sports Traumatology, knee surgery and arthroscopy. COVID-19 ESSKA guidelines and recommendations for resuming elective surgery. Available in: https://cdn.ymaws.com/www.esska. org/resource/resmgr/covid-19/COVID-guidelines-Q\&A.pdf. 